\title{
Comparison of analgesic efficacy of oxycodone and fentanyl after total hip replacement surgery
}

\author{
H. Y. Kang, S. W. Park, H. H. Woo \\ Kyung Hee University Hospital - Seoul (South Korea)
}

Background and Goal of Study : Total hip replacement (THR) is often accompanied by severe postoperative pain. There are several studies that oxycodone has sufficient analgesic effect and somewhat higher, but tolerable side effects compared with fentanyl as adjunctive drug in treating both acute and chronic moderate to severe post-operative pain without ceiling effects. However most of studies expected visceral pain relief about $k$ receptor effect. In this study, we designed to study whether oxycodone can be an effective alternative of fentanyl at early post-operative pain management in orthopedic surgery.

Materials and Methods : Twenty patients were involved in randomized control trial. Anesthesia was induced with $1-2 \mathrm{mg} / \mathrm{kg}$ propofol and $0.8 \mathrm{mg} / \mathrm{kg}$ rocuronium. Desflurane (5-7 vol\%) was used with air (50\%) and oxygen (50\%) mixture for maintenance of anesthesia. Remifentanil was infused 1.5-5.0 $\mu \mathrm{g} / \mathrm{min}$. Analgesic agents were administered at 20 min before the end of surgery; $50 \mu$ fentanyl $(F)$ group $(n=10)$ and $4 \mathrm{mg}$ oxycodone $(O)$ group $(n=10)$. Numeric rating scale (NRS,0-10;0 = no pain and $10=$ worst possible pain) of postoperative pain at post anesthetic care unit (PACU) were measured. All patients had intravenous patient-controlled analgesia (IV PCA) with $15 \mu \mathrm{g} / \mathrm{kg}$ fentanyl for 2 days. $(0.31 \mu \mathrm{g} / \mathrm{kg} / \mathrm{h})$. Additional intravenous fentanyl bolus was used instead of pushing PCA bolus button at ward. It was monitored that additional analgesic agent was administered during 0-6, 6-12, 12-24, and 24-48 $\mathrm{h}$ after surgery. Mann Whitney $U$ test was performed to compare continuous variables between the two groups. $P$ value less than 0.05 was considered statistically significant.
Results and Discussion: There were no statistically significant differences between the two groups in patient age, weight, height, duration of surgery, and total remifentanil consumption $(P>0.05)$. NRS of group $O$ at PACU was lower than that of group $F(P<0.05)$. (Fig. 1) A lower proportion of patient's number was observed in Group O than Group F during 0-6 h; it is statistically significant $(\mathrm{P}<0.05)$. (Table 1)

Conclusion(s) : Oxycodone revealed lower NRS and lower fentanyl consumption during early postoperative period compare with fentanyl. Therefore, we conclude that oxycodone is more effective than fentanyl in acute phase of postoperative pain control. Oxycodone will be another analgesic agent at postoperative period in orthopedic surgery.

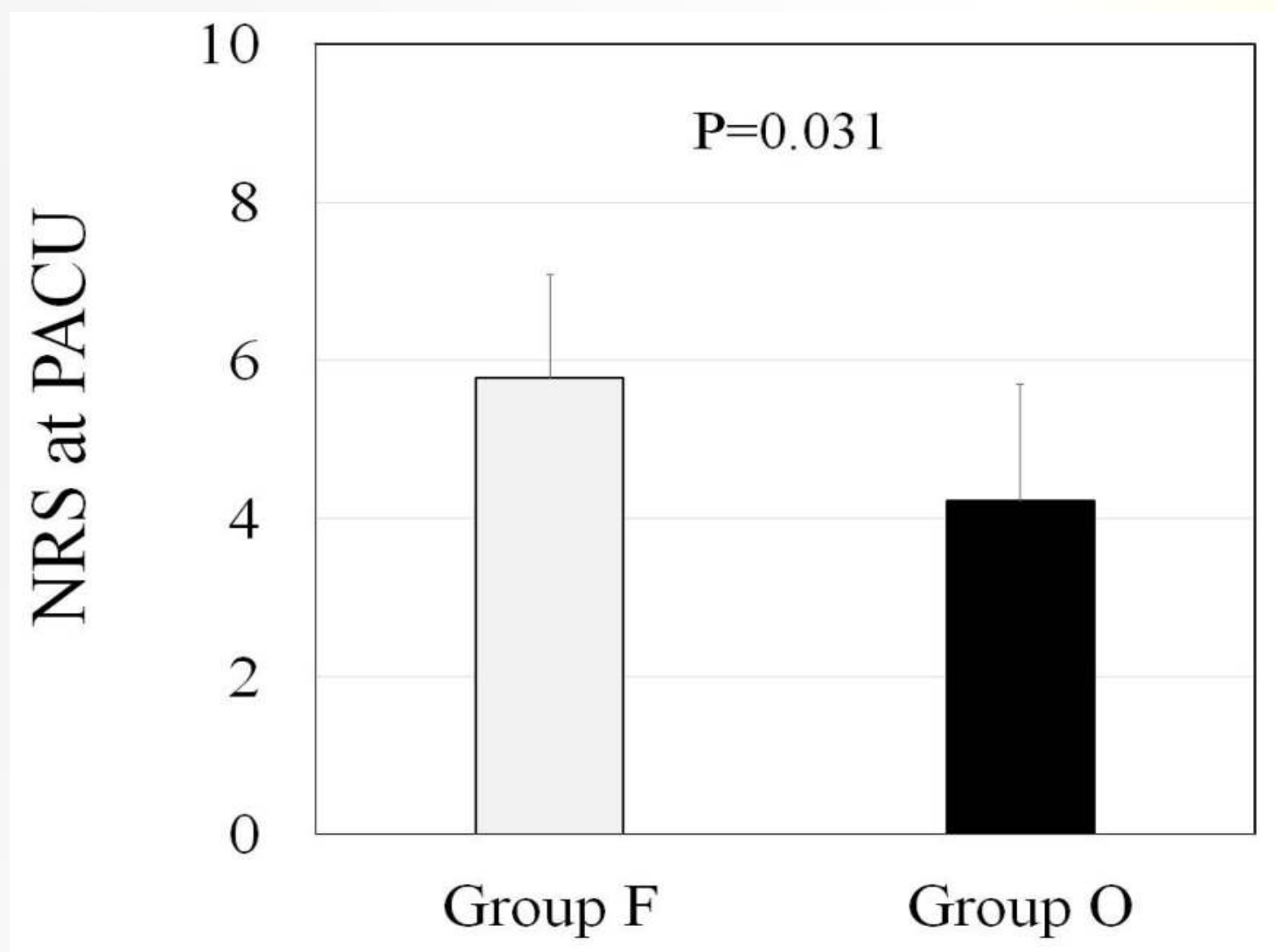

Fig. 1. NRS at PACU
Time after surgery $(h)$
Group F ( $=10)$
Group O (n=10)
P value

\begin{tabular}{|cccc|}
\hline $0-6$ & $9(90.0 \%)$ & $4(40.0 \%)$ & 0.012 \\
\hline $6-12$ & $1(10.0 \%)$ & $1(10.0 \%)$ & 0.500 \\
\hline $12-24$ & $2(20.0 \%)$ & $2(20.0 \%)$ & 0.500 \\
\hline $24-48$ & $1(10.0 \%)$ & $1(10.0 \%)$ & 0.500 \\
\hline
\end{tabular}

